\title{
USOS E ABUSOS DA HIPERVENTILAÇÃO NOS TRAUMATISMOS CRÂNIO-ENCEFÁLICOS GRAVES
}

\author{
FRANCISCO CARLOS DE ANDRADE* FRANCISCO CARLOS DE ANDRADE JR**
}

\begin{abstract}
RESUMO - É feita avaliação crítica sobre as diretrizes atuais e consequências da manobra de hiperventilação (MHV) na prevenção e tratamento da hipertensão intracraniana (HIC) que segue aos traumatismos crânioencefálicos (TCE) graves. O uso profilático da MHV deve ser evitado na fase aguda de TCE grave, a menos que se registrem altos valores de $\mathrm{O}_{2}$ no sangue venoso medido no bulbo jugular, ou para ganhar tempo quando o paciente apresenta evidentes sinais posturais de deterioração neurológica. A falta de resposta cerebrovascular à MHV para baixar a HIC significa que a barreira hemato-encefálica (BHE) está difusamente lesada. Então, a MHV pode ser utilizada como um crivo nos TCE graves, uma vez que a lesão da BHE atesta que os demais tratamentos disponíveis para combater a HIC (sedação, paralisia e diuréticos osmóticos) não funcionarão. Uma nova hipótese patogênica do edema encefálico traumático e abordagem terapêutica é apresentada.
\end{abstract}

PALAVRAS-CHAVE: traumatismo crânio-encefálico, terapêutica, fisiopatogenia, hiperventilação.

\section{Uses and abuses of the hyperventilation in severe traumatic brain injury}

ABSTRACT - A critical evaluatin was done about the guidelines and effects of the hyperventilation maneuver on prevention and treatment of increased intracranial pressure (ICP) that follows severe traumatic brain injury (TBI). The prophylatic use of hyperventilation should be avoided after severe TBI acute phase, unless high venous $\mathrm{O}_{2}$ values are recorded at jugular bulb blood $\left(\mathrm{SjO}_{2}\right)$, or to allow time when there are evidences of neurologic deterioration with posturing. The lack of cerebrovascular response to hyperventilation to low the ICP means that the blood brain barrier (BBB) function is extensively impaired. Then, hyperventilacion may be used as a screening therapeutic test in acute severe TBI, since BBB impairment is the pointer that other available clinical procedures for high ICP control (sedation, paralysis and osmotic diuretics) are not workable. A new pathogenetic hypothesis about traumatic brain edema and its therapeutic approach is presented.

KEY WORDS: head injury, therapy, hyperventilation, pathophysiology.

Após o projeto do Banco Nacional de Dados dos Comas Traumáticos nos Estados Unidos da América (EUA), 1983, se seguiram recomendações de hiperventilação precoce e agressiva na prevenção e tratamento da hipertensão intracraniana (HIC) aguda pós-traumática. Posteriormente, a análise dos resultados dos protocolos de tratamento dos traumatismos crânio-encefálico (TCE) graves realizados nos centros de atendimento especializados nos EUA e Europa não se notou qualquer benefício da manobra de hiperventilação (MHV) profilática e terapêutica no prognóstico vital e funcional desses pacientes.

Com a hipótese que o abuso generalizado e indiscriminado do uso da MHV nos protocolos de tratamento seguidos nos últimos 30 anos contribuiu para o malogro dos resultados das terapias adotadas nos TCE graves, resolveu-se revisar, criticar e divulgar as evidências científicas atuais sobre os efeitos da MHV sobre o metabolismo, mecanismo de auto-regulação da circulação encefálica e agravamento da HIC e edema cerebral isquêmico observado nesses pacientes. Pacientes com TCE

*Professor Titular de Neurologia do Centro de Ciências Médicas e Biológicas da Pontifícia Universidade Católica de São Paulo; **Neurocirurgião, Instituto de Ciências Neurológicas de São Paulo

Dr. Francisco Carlos de Andrade - Avenida General Osório 1112 - 18060-001 Sorocaba SP - Brasil.

E-mail: neto@andrade.com 
graves são ainda submetidos a MHV (de modo protocolar, descontrolado e despreocupado) em nosso meio, segundo dados da Sociedade Brasileira de Atendimento Integrado ao Trauma (SBAIT).

\section{O objetivo terapêutico da $M H V$}

A hipótese fisiopatogênica vigente nos anos 80 sobre a origem da HIC que segue aos TCE graves admitia que ocorria uma isquemia encefálica inicial seguida de hiperemia reativa, com consequente aumento do fluxo sanguíneo (FSC) e volume sanguíneo cerebral (VSC) ${ }^{1}$. Como o FSC é função da $\mathrm{PaCO}_{2}$, a $\mathrm{MHV}$ promove rápida redução do $\mathrm{FSC}$ e VSC, e tal procedimento era amplamente empregado na prevenção e tratamento da HIC pós-traumática ${ }^{2}$. Como o nível de hipocapnia desejado é facilmente obtido e mantido pela MHV, sua utilização se baseava na obtenção de um nível de redução da pressão intracraniana (PIC) considerada necessária e suficiente para permitir a manutenção adequada da pressão de perfusão cerebral (PPC). Deste modo, reduzindo-se o FSC e VSC ganha-se espaço intracraniano adicional para combater o efeito nocivo que o constrangimento progressivo que a HIC exerce sobre a PPC.

Simplesmente, com a MHV visa-se reduzir o FSC e VSC para evitar a nociva redução do FSC e VSC que acompanha os níveis mais graves de HIC pós-traumática, na esperança de poupar o encéfalo da falência de sua circulação e metabolismo. Assim, prevenindo e combatendo a HIC, a maioria dos clínicos assumia que a MHV beneficiaria a todos esses pacientes, sem nenhum efeito adverso.

\section{O FSC no TCE grave}

O único estudo clínico prospectivo e aleatório disponível mostrou que o prognóstico dos paciente com TCE graves foi pior aos 3 e 6 meses quando a MHV foi utilizada em relação ao grupo no qual foi omitida ${ }^{3}$. As pesquisas conduzidas sobre o assunto nos últimos 30 anos claramente evidenciam que o FSC durante os primeiros dias após o TCE é menor que a metade daquele encontrado nos indivíduos normais ${ }^{4-13} \mathrm{e}$ existe portanto um risco efetivo da MHV provocar isquemia encefálica nesses pacientes ${ }^{14}$. Existem evidências histopatológicas de isquemia cerebral encontradas em $90 \%$ das vítimas que morrem após TCE graves ${ }^{15-17}$

\section{$O$ efeito da MHV nos encéfalos normais}

A MHV pode aumentar a $\mathrm{PaO}_{2}$, mecanismo acreditado por muitos como responsável pela queda imediata observada na pressão intracraniana (PIC) elevada ${ }^{18-21}$. Alcançado um grau de hipocapnia moderada $\left(\mathrm{PaCO}_{2} \geq 27-30\right.$ torr $\left.\leq\right)$, já desvia-se a curva de dissociação da hemoglobina (Bohr), tornando a disponibilidade de $\mathrm{O}_{2}$ capilar menor e a avidez tissular cerebral por $\mathrm{O}_{2}$ maior. Disso resulta que a diferença arteriovenosa de $\mathrm{O}_{2}\left(\mathrm{DAVO}_{2}\right)$ se amplia, havendo menor saturação venosa de $\mathrm{O}_{2}\left(\mathrm{SjO}_{2}\right)$ ou seu parâmetro oposto, maior dessaturação de $\mathrm{O}_{2}$ no sangue venoso que deixa imediatamente o encéfalo (bulbo jugular) (Fig 1).

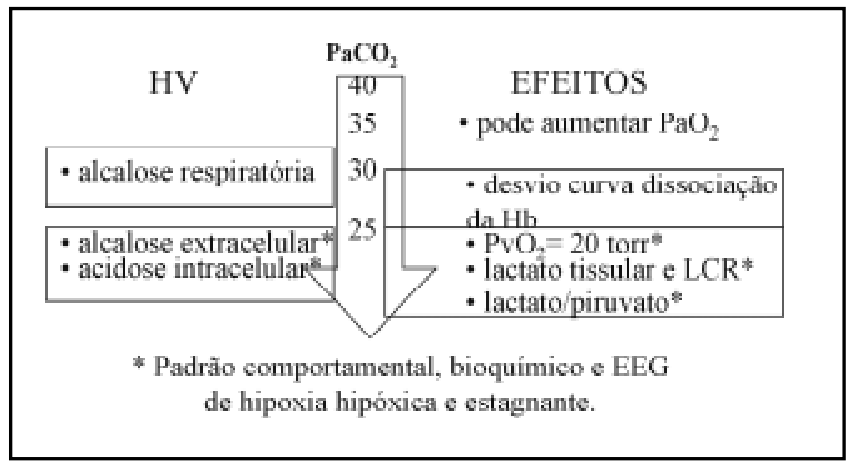

Fig 1. Fisiologia da HV. 


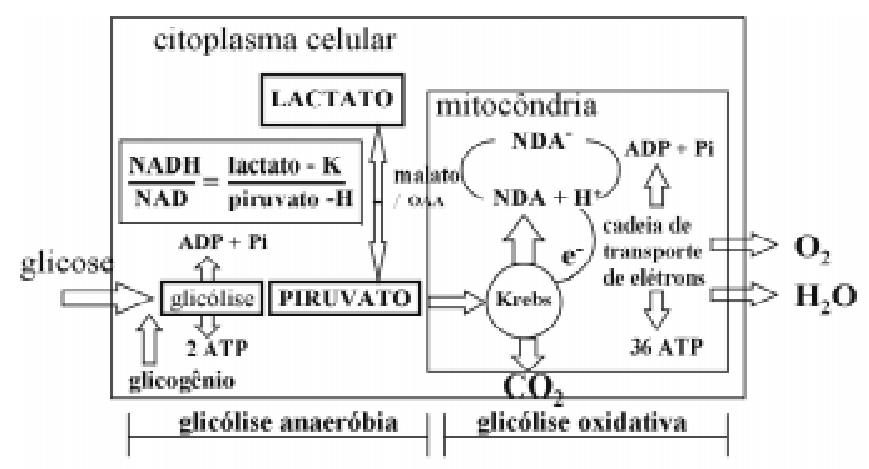

$\mathrm{H}^{+}$, ion hidrogênio ativo; $\mathrm{Pi}$, fosfato inorgânico; $\mathrm{NADH}, \mathrm{NAD}^{+}$, nicotinamida adenosina dinucleotídeo reduzida e oxidada; OAA, ácido oxalacético; é, elétron.

Fig 2. Metabolismo da glicose no tecido cerebral.

Atingindo $\mathrm{PaCO}_{2}$ de 25 torr, a extração de oxigênio pelo tecido encefálico perfundido $\left(\mathrm{DAVO}_{2}\right)$ é ainda maior e neste nível de hipocapnia a pressão venosa de $\mathrm{O}_{2}\left(\mathrm{PvO}_{2}\right)$ já atinge 20 torr no bulbo jugular, e é seguida de alteração comportamental (torpor) e ondas lentas no eletrencefalograma. Tais parâmetros atestam que a demanda metabólica tissular encefálica por $\mathrm{O}_{2}$ já não está sendo atendida pelo FSC correspondente, devido hipóxia.

Com hipóxia tissular cerebral há aumento da glicólise anaeróbica, e a energia disponível às células é insuficiente para manter as funções de suas membranas, e a passagem do piruvato às mitocôndrias ao ciclo de Krebs se restringe, acumulando-o no citoplasma. Deste modo, o piruvato, cuja concentração está em equilíbrio constante com o lactato no citoplasma, ao elevar-se acaba por aumentar a concentração de ácido láctico neste, acidificando-o (Fig 2).

A relação $\mathrm{NADH} / \mathrm{NAD}^{+}$seguirá a relação lactato/piruvato citoplasmático, e privado de oxigênio o $\mathrm{NAD}^{+}$não se oxida e o potencial redox do sistema cai, o que provoca a parada do fluxo de elétrons no sistema de transporte das membranas. Isto, aumentará ainda mais a concentração de íons $\mathrm{H}^{+}$citoplásmico, ou seja, agrava a lactoacidose hipoxêmica do tecido (Fig 3).

$$
\text { Hipoxia }=\frac{\mathrm{NADH}}{\mathrm{NAD}^{+}}=\frac{\text { lactato }}{\text { piruvato }} \cdot \frac{\mathrm{K}}{\mathrm{H}^{+}}=15
$$

Fig 3. Estado redox citoplasmático.

A lactoacidose é, portanto, a contrapartida metabólica da hipóxia celular e seguramente um parâmetro bioquímico do desencontro entre FSC e necessidade metabólica neuronal.

Como contraprova, temos que todas as alterações bioquímicas, comportamentais e electrencefalográficas observadas na hipocapnia moderada e agressiva são prontamente revertidas pela oxigenação hiperbárica.

Os efeitos da MHV sobre a circulação e metabolismo encefálico, promovendo de um lado hipocapnia, alcalose extracelular e vasoconstrição, acaba provocando lactoacidose intracelular que suscitaria por outro lado vasodilatação e aumento do FSC (Fig 4). 


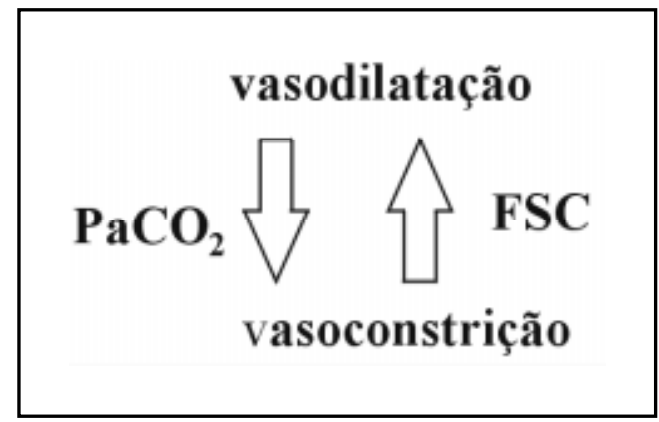

Fig 4. Mecanismo de retro-alimentação vasomotora. A hipocapnia provoca redução do FSc e hipóxia e lactoacidose que promove vasodilatação.

Este é o conhecido mecanismo bioquímico de retroalimentação da homeostase do FSC. Portanto, a MHV provoca alterações hipóxicas tissulares, acidose intra e extracelular (no encéfalo e líquor) dos indivíduos normais, e se moderada e prolongada $\left(\mathrm{PaCO}_{2} \geq 27\right.$ a 30 torr $\left.\leq\right)$ ou se agressiva e fugaz ( $\mathrm{Pa} \mathrm{CO} 2 \leq 25$ torr) desconecta o mecanismo de auto-regulação fisiológica da microcirculação encefálica, e desse modo, seria então uma manobra intrinsecamente iatrogênica, mesmo nos encéfalos normais $^{21,22}$.

A intensidade da resposta e duração da MHV sobre o FSC também foi estudada em voluntários sadios: 30 minutos após atingido $\mathrm{PaCO}_{2}$ entre 15-20 torr houve declínio de 40\% no FSC, e quatro horas após houve aumento do FSC acima de $90 \%$ do original e quando o $\mathrm{PaCO}_{2}$ inicial foi restaurado houve rebote de $31 \%$ no $\mathrm{FSC}^{23}$. Considerando adicionalmente os dados gerados pelas pesquisas básicas sobre o assunto neste últimos 30 anos, temos o que segue:

1. O FSC é baixo na fase aguda dos TCE graves - O FSC é muito baixo durante as primeiras 24 horas após o trauma e se restabelece progressivamente nos 3 dias subsequentes, exceto nos pacientes que desenvolvem HIC incontrolável e morrem ${ }^{3,6,8-12}$. O FSC é tipicamente menor que 30 $\mathrm{ml} / 100 \mathrm{~g} / \mathrm{min}$ durante as primeiras 8 horas após o trauma, e pode ser menor que $20 \mathrm{ml} / 100 \mathrm{~g} / \mathrm{min}$ durante as primeiras 4 horas após o TCE grave $e^{4-7,12,13}$. Ainda mais, nos TCE graves o FSC é baixo em pacientes com hematomas subdurais, lesões difusas e hipotensão arterial sistêmica, aumentado naqueles com hematomas epidurais e com tomografias computadorizadas de crânio (TCC) normais ${ }^{24-27}$. Existe correlação direta entre FSC, escala de coma de Glasgow (ECG) e prognóstico somente nas primeiras 48 horas após o TCE ${ }^{4,9}$. A alteração no FSC não se correlaciona necessariamente com uma mudança da PIC, e em alguns casos aumento no FSC causa um decréscimo na PIC ${ }^{14,19}$.

2. Quando o FSC é baixo a DAVO2 é alta - Existe relação inversa entre a $\mathrm{DAVO}_{2}$ e os valores do FSC durante as primeiras 24 horas após o TCE grave, exceto em paciente com hematomas subdurais $^{4,10,12,13,27,29}$ e DAVO $_{2}$ maiores que 9 vol \% provavelmente indicam isquemia cerebral ${ }^{14,19}$.

3. Quando o FSC é baixo a $\mathrm{SjO}_{2}$ é baixa - A saturação venosa de $\mathrm{O}_{2}$ no bulbo jugular $\left(\mathrm{SjO}_{2}\right)$ é normalmente maior que $50 \%$, e valores menores são considerados como índices de dessaturação. Episódios profundos e prolongados de dessaturação nos TCEs graves são associados com péssimo prognóstico ${ }^{29,30}$. A dessaturação de $\mathrm{O}_{2}$ no sangue venoso é frequentemente encontrada com FSC baixo $^{30}$.

4. A hipocapnia está associada com dessaturação venosa de $\mathrm{O}_{2}^{21-23,29-31}$

5. A MHV nos TCE graves provoca isquemia - Para examinar a possibilidade da MHV produzir isquemia cerebral significativa, o FSC regional (FSrC) e reatividade vascular foram medidos antes e após MHV usando TCC contrastada com Xe em 12 pacientes com lesões encefálicas agudas póstraumáticas $^{32}$. Em 5 pacientes a MHV induziu isquemia em regiões encefálicas aparentemente normais; em 3 pacientes, áreas de perfusão de luxo tornaram-se isquêmicas durante a MHV; em 3 pacientes 
com áreas com lesões isquêmicas moderadas, estas áreas se tornaram ainda mais isquêmicas. A maioria dos pacientes mostrou mais que um tipo de reatividade vascular. Estes achados documentam a isquemia induzida pela MHV na fase aguda dos TCEs e demonstram que este fenômeno afeta ambas, áreas lesadas e aparentemente intactas do encéfalo ${ }^{32}$.

$\mathrm{O}$ 'FSR cortical ( $\mathrm{FSrCC}$ ) foi continuamente monitorado através do método de difusão térmica em 56 pacientes com TCE graves ${ }^{33}$. Os dados acumulados de 37 pacientes foram considerados adequados ao estudo ( 21 hematomas subdurais, 10 contusões cerebrais, 4 hematomas epidurais e 2 HIC). Dentre estes, em 15 pacientes a monitoração iniciou-se dentro das primeiras 8 horas pós-trauma. Um terço dos pacientes comatosos nas primeiras 8 horas tinham FSrcC de $18 \mathrm{ml} / 100 \mathrm{~g} / \mathrm{min}$ ou menos, achados consistentes com os relatos prévios de isquemia no período pós-traumático precoce. As medidas foram similares nos pacientes com escores na ECG de 3 a 7, assim como naqueles com 8 ou mais. Nos pacientes com mau prognóstico, as medidas do $\mathrm{FSrcC}$ não se alteraram significativamente durante a evolução e naqueles com melhor prognóstico as medidas do FSrcC tiveram normalização progressiva e significativa. Quando o manejo terapêutico foi baseado estritamente no combate a HIC foram encontrados vários exemplares de tratamento inapropriado. Por ex., em hiperemia e HIC tratados com Manitol $^{\circledR}$, houve aumento adicional do FSrcC; e em HIC com FSC baixo tratados com MHV houve piora da severidade da isquemia. O registro contínuo do $\mathrm{FSrcC}$ em pacientes com TCE oferece nova visão terapêutica e prognóstica no manejo desses pacientes ${ }^{33}$.

O estudo de 16 pacientes pela tomografia por emissão de pósitrons (PET) com evidente enfarte hemisférico, registrada em média 23 horas após o início dos sintomas, mostrou que o FSC foi $16,7 \pm 7,95 \mathrm{ml} / 100 \mathrm{~g} / \mathrm{min}$ no centro do enfarto e de $31,0 \pm 10,65 \mathrm{ml} / 100 \mathrm{~g} / \mathrm{min}$ na zona imediatamente adjacente ao enfarto. Tais enfartos isquêmicos são ocorrências comuns quando o FSC cai abaixo de 15 e $20 \mathrm{ml} / 100 \mathrm{~g} / \mathrm{min}$ nos TCE graves $^{34}$.

O resultado do único estudo prospectivo, até agora disponível, no qual 77 pacientes com TCE foram aleatoriamente endereçados ao grupo de tratamento com $\mathrm{MHV}$ profilática por 5 dias $\left(\mathrm{PaCO}_{2}=\right.$ $25 \pm 2$ torr), e no outro grupo tratado para manter o $\mathrm{PaCO}_{2}=35 \pm 2$ torr por 5 dias após trauma, encontrou que aos 3 e 6 meses, pacientes com escores motores iniciais de 4-5 na ECG no grupo da MHV tiveram pior prognóstico que o grupo "normocápnico". Após 1 ano, não havia diferença significativa entre os 2 grupos, mas, isto poderia ser atribuído a erro estatístico do tipo II, por existirem muito poucos pacientes disponíveis para análise no seguimento após $1 \mathrm{ano}^{3}$.

Concluiu-se, em 1995, que a MHV não é mais recomendada como uma manobra terapêutica ou profilática de primeira linha para combater a HIC pós TCE grave, por ser intrinsecamente isquemiante e iatrogênica nos TCE graves ${ }^{35}$.

\section{DISCUSSÃO}

Logo, existe numerosa base de dados científicos consistentes e considerável evidência clínica de que a MHV provoque agravamento do prognóstico dos pacientes com TCE graves. Nestes tempos, a MHV somente seria indicada na eventualidade de que HIC pós-traumática fosse causada predominantemente pelo aumento do FSC e VSC (inflação, inchaço cerebral), e nesses casos a $\mathrm{SjO}_{2}$ seria alta $(>75 \%)$. Determinando-se quantidade de $\mathrm{O}_{2}$ tomada pelo tecido cerebral $\left(\mathrm{DAVO}_{2}, \mathrm{SjO}_{2}\right)$ nesses casos, teríamos parâmetros para assegurar que o tecido cerebral traumatizado poderia suportar uma redução adicional do seu suprimento de $\mathrm{O}_{2}$ provocado pela vasoconstrição hipercápnica da $\mathrm{MHV}$ visando reduzir sua documentada HIC, pelo registro contínuo de sua PIC. Mas, por outro lado, isto também atesta que existe um desencontro entre necessidades do metabolismo cerebral e do fluxo sanguíneo capilar, isto é, o oxigênio disponível no FSC não é aproveitado pelo encéfalo, a ponto de sobrar no sangue venoso que o atravessa e o deixa, com $\mathrm{DAVO}_{2}$ baixa e $\mathrm{SjO}_{2}$ alta. Ainda mais, a distinção clínica e radiológica entre inflação (ou inchaço) e edema cerebral não é fácil apenas com esses recursos, e encontrar casos clínicos puros de uma forma ou de outra é ainda muito mais difícil.

Isto posto, a dificuldade prática de indicação profilática e terapêutica da MHV nos TCE é evidente, pois sem o conhecimento prévio desses parâmetros bioquímicos metabólicos $\left(\mathrm{DAVO}_{2}\right.$, 
$\mathrm{SjO}_{2}$ ) e instrumentais como PIC, PPC nas primeiras 24 horas, a MHV moderada e continuada ou agressiva e temporária nesses pacientes é contraproducente e frequentemente danosa, excetuando talvez, por absoluta falta de alternativas, aqueles pacientes que se apresentam com sinais de herniação intracraniana clinicamente evidente, visando ganhar-se tempo para outras providências, como detecção de uma afeção cirúrgica pela TCC.

\section{Sobre a fisiopatogenia da redução do FSC observada nos TCE graves}

Existem três hipóteses fisiopatogênicas disponíveis para explicar a queda inicial do FSC observada nos TCE graves:

a) Pode ser que o coma que define os TCE graves provoque por si só depressão do FSC 4,9,14,28. Mas, na hipótese da redução do FSC observada na fase aguda dos TCE graves ser consequente a baixas necessidades metabólicas observadas nos estados de coma em geral, a $\mathrm{TMCO}_{2}, \mathrm{DAVO}_{2}$ nesses casos deveria ser normal e não haveria dessaturação de $\mathrm{O}_{2}$ medido no bulbo jugular $\left(\mathrm{SjO}_{2}\right)$. Os dados básicos disponíveis demonstram que, na fase precoce dos TCE graves, há aumento das necessidades metabólicas encefálicas e a PPC é frequentemente inadequada, por hipotensão arterial, hipóxia, anemia, e ocorre acidose metabólica sistêmica e encefálica ${ }^{21-23}$.

b) Outra hipótese é que o FSC esteja baixo na fase aguda dos TCE graves por diminuição da PPC consequente ao aumento da PIC ocasionada pelo trauma ${ }^{1,2}$. Os dados básicos disponíveis indicam que a variação do FSC observado não se relaciona necessariamente com alteração na PIC e, em alguns casos, o aumento do FSC causa concomitantemente diminuição na PIC ${ }^{19,25}$. Mesmo na ausência de HIC o FSC é baixo nos TCE graves ${ }^{16}$ devido ao vasosespasmo pós-traumático, como documentado em $40 \%$ desses pacientes ${ }^{36}$. O FSC é aumentado naqueles com hematomas epidurais (evidências de herniações e HIC) e com TCC normais (sem evidências de herniações e HIC) ${ }^{24-27}$.

c) A nova hipótese é que a queda do FSC é devida a lesão primária da barreira hematoencefálica (BHE) provocada pelo TCE grave e consequente perda do controle do volume cerebral (edema e inchaço ${ }^{37,38}$. Como o metabolismo cerebral conduz e não é conduzido pelo FSC, e como o metabolismo cerebral está seguramente aumentado na fase aguda do TCE grave, o desacoplamento do FSC das necessidades metabólicas cerebrais somente poderia significar que há lesão da BHE.

\section{A MHV pode testar o estado de reatividade vascular cerebral}

Como a MHV reduz a PIC por provocar vasoconstrição e subsequente redução no FSC e VSC, tal manobra seria um recurso prático na aferição da reatividade vascular encefálica, ou seja, o estado funcional da $\mathrm{BHE}^{18}$.

Assim, a resposta da PIC à MHV seria precioso parâmetro clínico que atestaria o estado de reatividade vascular encefálica, e a ausência de resposta da HIC significaria, e objetivaria na prática, a perda da função seletiva da BHE e prognosticaria a resposta a outras formas de terapia convencionais (sedação, paralisia e diuréticos osmóticos) na HIC aguda dos TCE graves.

Finalmente, cerca de 50\% dos pacientes com TCE graves se apresentam com hiperventilação "espontânea" na admissão (devido a acidose extracelular encefálica e do líquor), ${ }^{21,22}$ e é recomendado que sejam submetidos a paralisia muscular e ventilação normocápnica para evitar-se o agravamento do esperado baixo FSC inicial.

\section{As novas tendências}

A tendência atual é que o cuidado do paciente com TCE grave se desloque progressivamente para o cuidado médico dos intensivistas (idealmente, anestesiologistas), ficando o paciente dependente do neurocirurgião na eventual necessidade de indicação de tratamento cirúrgico.

A patogenia atual do edema cerebral pós-TCE se credita a perda do controle da permeabilidade e da regulação do volume de líquidos intra e extracapilares pelos seus endotélios especiais (junções estreitas) e consequente perda do controle do volume encefálico, gerada pela rotura da BHE que desregra os mecanismos que mantém o volume encefálico normal, induzindo a formação de edema, 
e pela lesão da BHE a circulação capilar do encéfalo estaria sujeita a lei que regula aos demais capilares sistêmicos (Starling).

Ensaio para avaliar nova terapia do edema pós-traumático é baseado no conceito de que a abertura da BHE perturba a regulação normal do volume encefálico, induzindo a formação do edema. Isto significa que os fluxos de fluídos transcapilares serão controlados pelas pressões hidrostáticas (PHc) e coloidosmóticas dos capilares (PCOc), mais que a pressão cristaloidosmótica. Se assim for, a terapia do edema encefálico deve incluir a redução da PHc e preservar a PCOc normal. Em 11 pacientes comatosos devido a TCE grave e edema encefálico, HIC e resposta cerebrovascular abolida, foi testada sua resposta pela $\mathrm{MHV}^{37,38}$. Para reduzir a $\mathrm{PH}$ desses pacientes foi dada terapia hipotensora arterial (antagonistas $\beta$ 1, metoprolol, e agonistas $\alpha 2$, clonidina) e um potencial vasoconstritor précapilar associado (dihidroergotamina). O último pode também diminuir o VSC através constrição da capacitância venosa. A PCOc foi mantida por infusão de albumina. O conceito implica na necessidade de um balanço de fluidos negativo com normovolemia preservada. Como resultado foi observado que a PIC diminuiu significativamente dentro de poucas horas de tratamento e a PPC ficou inalterada a despeito da queda da pressão arterial. Dos 11 pacientes, 9 sobreviveram com boa recuperação e incapacidade moderada, e 2 morreram. Isto foi comparado ao prognóstico de um grupo controle histórico com idêntico critério de entrada, usando terapia convencional do edema, em que a mortalidade/estado vegetativo/incapacidade funcional grave foi $100 \%$. Esses resultados indicam que a terapia deve focalizar o edema extracelular mais que o edema intracelular e que a isquemia não é o principal mecanismo original que dispara a formação do edema. Esta terapia é superior à convencional para prevenir herniações durante o período de recuperação da $\mathrm{BHE}^{37,38}$.

\section{Conclusões}

A MHV severa $\left(\mathrm{PaCO}_{2} \geq 25\right.$ torr) é danosa mesmo nos indivíduos normais, por provocar hipoxemia e acidose metabólica e se, continuada, acaba por desacoplar o desejável mecanismo bioquímico fisiológico de auto-regulação circulatória cerebral.

As medidas do FSC, no período mediato ao TCE graves, demonstram que o FSC é baixo, e enfaticamente sugerem que nas primeiras horas após trauma os valores absolutos aproximam-se daqueles consistentes com isquemia. A MHV, nesse período, reduzindo o FSC ainda mais, e nem sempre causando uma redução consistente da PIC para melhorar a PPC, pode provocar a perda do mecanismo de auto-regulação circulatória encefálica.

A MHV profilática e terapêutica continuada devem ser evitadas nos primeiros 5 dias após um TCE grave, e especialmente proscrita durante as primeiras 24 horas.

A ausência de resposta de redução da PIC pela manobra de HV indica que a BHE está difusa ou extensamente lesada, e que as outras medidas terapêuticas disponíveis para combater a HIC (sedação, paralisia e diuréticos osmóticos) e dependentes de uma BHE íntegra serão infrutíferas.

Enquanto nos TCE graves ainda não há falência da BHE, e somente nos casos comprovados de HIC por aumento exclusivo do VSC, e especificamente e seletivamente nessa situação, haveria ainda indicação de MHV terapêutica, embora não exista consenso e evidências científicas de sua eficácia.

O paciente com TCE grave que é admitido com hiperventilação "espontânea" deve ser submetido a paralisia muscular e respiração controlada para corrigi-la.

A MHV permanece ainda como um recurso heróico no TCE graves com sinais evidentes de herniação (decorticação e descerebração) para se ganhar tempo para afastar uma patologia cirúrgica que possa revertê-la imediatamente.

\section{REFERÊNCIAS}

1. Graham DI, Adams JH, Gennarelli TA. Pathology of brain damage. In Cooper PR (ed). Head injury. Baltimore: Williams \& Wilkins, 1982;5:72-88.

2. Lundberg N, Kjallquist A, Bien C. Reduction of increased intracranial presssure by hyperventilation. Acta Psychiatr Scand 1959;34:4-64. 
3. Muizelaar JP, Marmarou A, Ward JD, et al. Adverse effects of prolonged hyperventilation in patients with severe head injury: a randomized clinical trial. J Neurosurg 1991;75:731-739.

4. Bouma GJ, Muizelaar JP, Choi SC, et al. Cerebral circulation and metabolism after severe traumatic brain injury: the elusive role of ischemia. J Neurosurg 1991;75:685-693.

5. Bouma GJ, Muizelaar JP, Stringer WA, et al. Ultra early evaluation of regional cerebral blood flow in severe head injured patients using xenon enhanced tomography. J Neurosurg 1992;77:360-368.

6. Cruz J. Low clinical threshold for cerebral blood flow in severe acute brain trauma: case report. J Neurosurg 1994;80:143-147.

7. Fieschi C, Battistini N, Beduschi A, et al. Regional cerebral blood flow and intraventricular pressure in acute head injuries. J Neurol Neurosurg Psychiatry 1974;37:1378-1388.

8. Jaggi JL, Obrist WD, Gennarelli TA, et al. Relationship of early cerebral blood flow and metabolism to outcome in acute head injury. J Neurosurg 1990;72:176-182.

9. Marion DW, Darby J, Yonas H. Acute regional cerebral blood flow changes caused by severe head injuries. J Neurosurg, 1991;74:407-414.

10. Muizelaar JP, Marmarou A, Salles AA, et al. Cerebral blood flow and metabolism in severely head-injured children: Part. I: Relationship with GCS score, outcome, ICP, and PVI. J Neurosurg 1989;71:63-71.

11. Robertson CS, Clifton GL, Grossman RG, et al. Alterations in cerebral availability of metabolic substrates after severe head injury. J Trauma 1988;28:1523-1532.

12. Salvant JB, Muizelaar JP. Changes in cerebral blood flow and metabolism related to the presence of subdural hematoma. Neurosurgery 1994:80;324-327.

13. Schroder ML, Muizelaar JP, Kuta AJ. Documented reversal of global ischemia immediately after removal of an acute subdural hematoma. Neurosurgery 1994;80:324-327.

14. Obrist WD, Langfitt TW, Jaggi JL, et al. Cerebral blood flow and metabolism in comatose patients with acute head injury. J Neurosurg 1984;61:241-253.

15. Graham DI, Adams JH. Ischaemic brain damage in fatal head injuries. Lancet 1971;1:265-266.

16. Graham DI, Lawrence $\mathrm{AE}$, Adams $\mathrm{JH}$, et al. Brain damage in fatal non-missile head injury without high intracranial pressure. J Clin Pathol 1988;41:34-37.

17. Ross DT, Graham DI, Adams JH: Selective loss of neurons from the thalamic reticular nucleus following severe human head injury. J Neurotrauma 1993;10:151-165.

18. Cold GE, Christensen MS, Schmidt K. Effect of two levels of induced hypocapnia on cerebral autorregulation in acute phase of head injury coma. Acta Anesthesiol Scand 1981;25:397-401.

19. Bouma GJ, Muizelaar JP. Relashionship between cardiac output and cerebral blood flow in patients with intact and with impaired autoregulation. J Neurosurg 1990;73:368-374.

20. Becker DP, Miller, Ward JD, et al. The outcome from severe head injury with early diagnosis and intensive management. J Neurosurg 1997;47:491-502.

21. Gordon E, Pontén U. The non-operative treatment of severe head injuries. In Vinken PJ, Bruyn GW (eds). Handbook of clínical neurology. Amsterdam: North-Holland, 1976;24:599-626.

22. Siesjo B. Latic acidosis in brain: occurrence, triggering mechanism and pathological importance. Ciba Found Symp, 1982;87:77-100.

23. Reichle ME, Posner JB, Plum F. Cerebral blood flow during and after hyperventilation. Arch Neurol 1970;23:394-403.

24. Marchall LF, Smith RW, Shapiro HM. The outcome with aggressive treatment in sereve brain injuries: I. The significance of intracranial pressure monitoring. J Neurosurg 1979;50:20-25.

25. Miller JD. Head injury and brain ischaemia: implications for therapy. Br J Anaesth 1985;57:120-130.

26. Cold GE. The relationship between cerebral metabolic rate of oxygen and cerebral blood flow in acute phase of head injury. Acta Anesthesiol Scand 1986;30:453-457.

27. Narayan RK, Kishore PRS, Becker DP, et al. Intracranial pressure: To monitor or not monitor. J Neurosurg 1982;56:650-659.

28. Obrist WD, Gennarelli TA, Segawa H, et al. Relation of cerebral blood flow to neurological status and outcome in headinjured patients. J Neurosurg 1979;51:292-300.

29. Cruz J. On-line monitoring of global cerebral hypoxia in acute brain injury: relationship to intracranial hypertension. J Neurosurg, 1993;79:228-233.

30. Scheinberg M, Kanter MJ, Robertson CS, et al. Continuos monitoring of jugular venous oxygen saturation in head-injured patients. J Neurosurg 1992;76:212-217.

31. Cruz J, Miner ME, Allen SJ, et al. Continuous monitoring of cerebral oxygenation in acute brain injury: assessment of cerebral hemodynamic reserve. Neurosurgery, 1991;29:743-749.

32. Stringer WA, Hasso NA, Thompson JR, et al. Hyperventilation-induced cerebral ishemia in patients with acute brain lesions: demonstration by xenon-enhanced CT. Am J Neuroradiol 1993;14:475-484.

33. Sioutos PJ, Orozco JA, Weinand ME, et al. Continuous regional cerebral cortical blood flow monitoring in head-injured patientes. Neurosurgery 1995;36;943-950.

34. Heiss WD, Huber M, Fink GR, et al. Progressive derangement of peri-infarct viable tissue in ischemic stroke. J Cereb Blood Flow Metab 1992;12:193-203.

35. Marion DW, Firlik A, McLaughlin MR. Hyperventilation therapy for severe traumatic brain injury. New Horiz 1995;3:439-447.

36. Weber M, Grolimund P, Seiler RW. Evaluation of posttraumatic cerebral blood flow velocities by transcranial Doppler ultrasonography. Neurosurgery 1990;27:106-112.

37. Grände PO, Nordström, Asgeirsson B. Current alternative therapy of pos-traumatic edema. Nord Med 1994;5:157-159.

38. Asgeirsson B, Grände PO, Nordström CH. A new therapy of pos-trauma brain oedema based on haemodynamic principles for brain volume regulation. Intensive Care Med 1994;20:260-267. 